\title{
Radiation Impedance of an Array of Circular Capacitive Micromachined Ultrasonic Transducers in Collapsed State
}

\author{
Alper Ozgurluk, Student Member, IEEE, Abdullah Atalar, Fellow, IEEE, \\ Hayrettin Koymen, Senior Member, IEEE, Selim Olcum, Member, IEEE \\ Bilkent University, Electrical and Electronics Engineering Department, Ankara, Turkey
}

\begin{abstract}
Radiation impedance is one of the important parameters in designing efficient and wideband capacitive micromachined ultrasonic transducer (CMUT) arrays. It determines how much acoustical power is generated in the surrounding medium given the membrane motion. Recently, considerable effort has been put to characterize the radiation impedance of CMUT arrays in conventional uncollapsed regime. However, the radiation impedance of an array of CMUT cells in collapsed state has not yet been investigated. To calculate the array radiation impedance in collapse mode, we first calculate the radiation impedance of a single cell CMUT. For the array case, the mutual impedances between the neighboring cells must also be taken into account. We consider an array of $7,19,37$, and 61 cells placed in a hexagonal pattern and try to determine the radiation impedance for different degrees of collapse. We find that in the collapsed case the peak radiation resistance value is reached at higher $k d$ values, where $k$ is the wavenumber and $d$ is the center to center cell spacing, compared to the uncollapsed regime.
\end{abstract}

\section{INTRODUCTION}

Capacitive micromachined ultrasonic transducers (CMUTs) have drawn attention due to wide bandwidth they present especially when immersed in water [1]. Two available tools for the design and optimization of CMUTs are finite element method (FEM) simulations and electrical equivalent circuit approach. The latter is preferable since FEM simulations are time consuming and require knowledge of the usage of complex simulation programs. Many different equivalent electrical models have been proposed for the conventional (uncollapsed) operation mode. After the advent of high output pressures offered by the deep-collapse mode, a new electrical equivalent circuit has been created taking into account the nonlinear effects present in this mode [2]. Although this model is successful in predicting the dynamic characteristics of CMUTs, it lacks a true model of radiation impedance in the collapsed state which specifies how much acoustical power to be generated in the medium given the membrane motion.

In this work, we investigate the radiation impedance of an array of collapsed CMUT cells starting from the radiation impedance of a single collapsed CMUT cell [3]. It is determined analytically up to the antiresonance frequency and the results are verified by FEM simulation. Then, we find the radiation impedance of a collapsed array placed in a hexagonal pattern for different number of cells in the array and different $b / a$ ratios, where $b$ and $a$ (Fig. 1) are the contact and

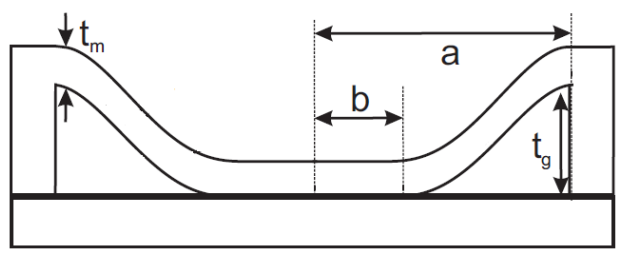

Fig. 1. Cross sectional view of a collapsed CMUT cell with radius $a$, contact radius $b$, thickness $t_{m}$, and gap height $t_{g}$.

membrane radii, respectively. The mutual impedance between the neighboring cells in the array is also taken into account to end up with accurate results. Finally, an electrical equivalent circuit for the radiation impedance of a collapsed array at an average $b / a$ ratio and for a limited $k d$ range is presented where $k$ is the wave number at the operating frequency and $d$ (Fig. 4) is the distance between two neighboring cells in the array.

\section{Single Cell Radiation Impedance}

\section{A. Method Overview}

The radiation impedance of a circular CMUT cell is defined as the ratio of the total power on the surface of the CMUT cell to the absolute square of the rms velocity of the membrane [4]:

$$
Z=\frac{P}{\left|V_{r m s}\right|^{2}}=\frac{\int_{S} p(r) v^{*}(r) d S}{\left[V_{r m s}\right]^{2}}
$$

where $p(r)$ and $v(r)$ are the pressure and the particle velocity, respectively, on the surface of the cell as a function of the radial distance and $S=\pi a^{2}$ is the surface area of the CMUT cell. The pressure generated on the surface of the membrane by the velocity profile of the CMUT cell must be known to find the radiation impedance. However, there is no available formula for the pressure generated by an arbitrary velocity profile. Therefore, we express the actual velocity profile, $v(r)$ as a linear combination of the functions given by [5] for which the generated pressures are known. These special functions are given as:

$$
v_{n}(r)=V_{r m s} \sqrt{2 n+1}\left(1-\left(\frac{r^{2}}{a^{2}}\right)\right)^{n} H(a-r)
$$




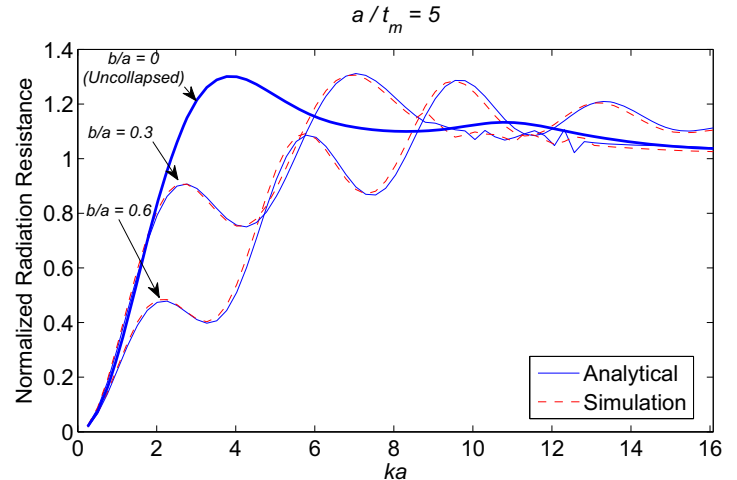

Fig. 2. Normalized radiation resistance for a single cell CMUT with $a / t_{m}=5$ in collapsed state for different $b / a$ ratios as a function of $k a$.

where $r$ is the radial distance, $a$ is the radius of radiator, $H$ is the unit step function, and $V_{r m s}$ is the rms velocity of the membrane as defined in [3].

We use these functions with $n=2,3,4,5$ and write down the actual velocity profile, $v(r)$, obtained from FEM simulation as:

$$
v(r)=\alpha_{2} v_{2}(r)+\alpha_{3} v_{3}(r)+\alpha_{4} v_{4}(r)+\alpha_{5} v_{5}(r)
$$

where $\alpha_{n}$ 's are real numbers. Having expressed the actual velocity profile in terms of the functions in (2), finding the total pressure on the surface of the CMUT cell is relatively easy thanks to the formula in [5] giving the pressure generated by each of the velocity profiles in (2). The total pressure on the surface can be written as a linear combination of the pressures generated by each of the velocity profiles with the same weighting coefficients, $\alpha_{n}$, in (3) :

$$
p(r)=\alpha_{2} p_{2}(r)+\alpha_{3} p_{3}(r)+\alpha_{4} p_{4}(r)+\alpha_{5} p_{5}(r)
$$

where $p(r)$ is the total pressure on the surface and $p_{n}(r)$ is the pressure generated by the velocity profile $v_{n}(r)$. Radiation impedance can be found by inserting (3) and (4) in (1).

\section{B. Single Cell Radiation Impedance}

The radiation resistance and reactance values normalized by $\rho S c$ for a single cell CMUT in collapsed state are shown in Figs. 2 and 3, respectively. The figures show that the radiation resistance and reactance for lower $k a$ values become smaller in collapse mode as compared to the conventional regime. In addition, as $b / a$ ratio increases, the radiation impedance at aforementioned $k a$ values keeps decreasing further. Also the radiation resistance and reactance values become more oscillatory with the increasing $b / a$ ratio. In the figures, even the glitches show the same behavior in some cases for both analytical and FEM results. This is due to the fact that both methods use the same velocity profile obtained from FEM simulation since there is no analytical expression available for the velocity profile of a collapsed CMUT cell.

The crucial point in calculating the radiation impedance of a radiator is the determination of the particle velocity distribution on the surface of the radiator, since the radiation impedance is only a function of the particle velocity distribution - pressure on the surface of the radiator is also a

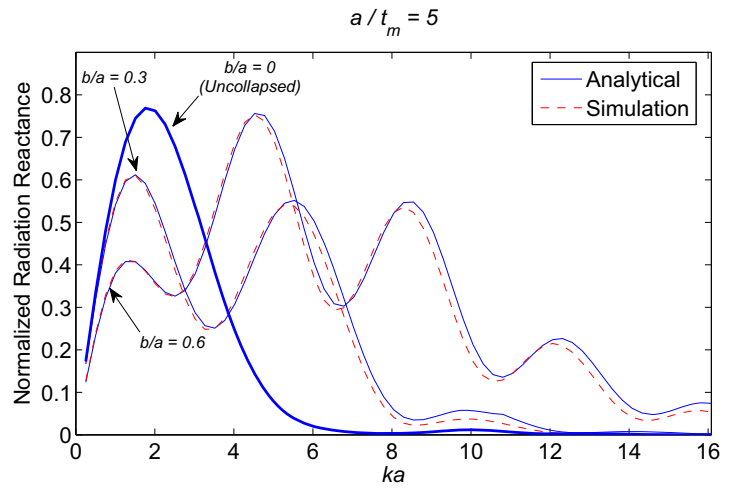

Fig. 3. Normalized radiation reactance for a single cell CMUT with $a / t_{m}=5$ in collapsed state for different $b / a$ ratios as a function of $k a$.

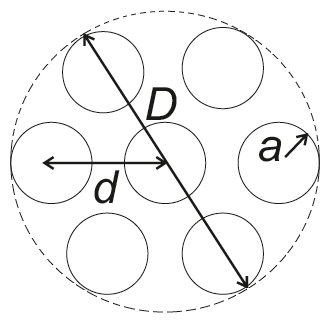

Fig. 4. Geometry of an array placed in a hexagonal pattern with 7 cells

function of the velocity profile- [5]. In the uncollapsed mode, such an expression is analytically available and every CMUT membrane obeys that normalized velocity profile as long as the operating frequency is not in the vicinity of its antiresonance. Using this velocity profile, only one radiation impedance result for the CMUT cells is obtained which is independent of any geometrical variables. Every CMUT membrane shows this radiation impedance characteristic as long as the operating frequency is not so close to the antiresonance. If the operating frequency approaches to the antiresonance, the velocity profile of the CMUT deviates from the given analytical expression and so also is the radiation impedance. However, such a readyto-use analytical formula for the particle velocity distribution on the surface of a CMUT cell is not available in the collapsed state, and therefore, we still perform FEM simulations to find $v(r)$ in the analytical calculation and include the membrane in the simulations. The most general radiation impedance in the collapsed case can be found using a thick membrane as a function of $b / a$ ratio as seen in Figs. 2 and 3 since the antiresonance frequency of a thick membrane is quite high.

\section{ARRAY RADIATION IMPEDANCE}

\section{A. Method Overview}

We consider an array of $7,19,37$, and 61 cells placed in a hexagonal pattern as shown in Fig. 4 for 7 cells configuration. In the case of an array of CMUT cells, each cell experiences an acoustic loading from the neighboring cells. Therefore, the mutual impedances between the cells must also be taken into account. The mutual impedance $Z_{i j}$ between the two CMUT cells is defined as the ratio of the power generated on surface 


\begin{tabular}{ccc}
\hline$b / a$ & Opt. a/d & Increase in Peak \\
\hline 0.25 & 0.47 & $2.75 \%$ \\
0.43 & 0.43 & $12.66 \%$ \\
0.50 & 0.41 & $20.13 \%$ \\
0.63 & 0.38 & $31.51 \%$ \\
\hline
\end{tabular}

TABLE I

OPTIMUM $a / d$ FOR DIFFERENT $b / a$ RATIOS AND PERCENT INCREASE IN PEAK RESISTANCE VALUE COMPARED TO CLOSE PACKED PLACEMENT

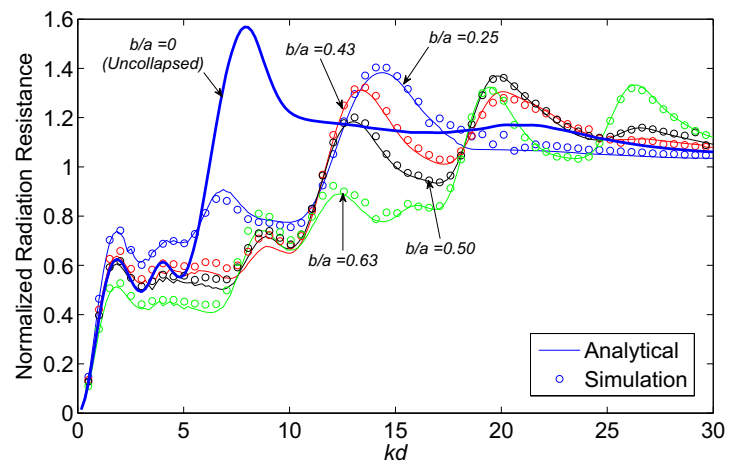

Fig. 5. Normalized radiation resistance for a 7-cell closely packed CMUT array in collapsed state for different $b / a$. For comparison, the radiation resistance in uncollapsed regime is also included.

of the $j$ th cell due to the pressure generated by the $i$ th cell to the product of the rms velocities of the $i$ th cell and the $j$ th cell [4]. Employing this definition $Z_{i j}$ can be written as:

$$
Z_{i j}=\sum_{n=2}^{5} \sum_{m=2}^{5} \alpha_{n} \alpha_{m} Z_{i j}^{n m}
$$

where $Z_{i j}^{n m}$ is the mutual impedance between the $i$ th and $j$ th transducers having the velocity profiles $v_{n}(r)$ and $v_{m}(r)$ in (2).

With such a definition, radiation impedance of two CMUT cells located in different tiers of the array might be different. Therefore, we must either determine the radiation impedance of an array by specifying the radiation impedance of an each layer separately or find a representative or an average radiation impedance for the whole array. We use the same representative radiation impedance definition for an array of circular CMUT cells given in [3].

\section{B. Array Radiation Impedance}

We present two sets of results for the radiation impedance of an array of circular CMUTs in collapsed state. In Figs. 5 and 6 , the number of cells in a closely packed array $(a / d=0.50)$ is kept constant at $N=7$ and the contact radius is varied. We find that the peak radiation resistance value is reached at higher $k d$ values in the collapse mode as compared to the uncollapsed mode. It is worth mentioning that this peak value is always smaller than that of the uncollapsed case. In addition, with the increasing contact radius, the radiation resistance becomes smaller for almost all values of $k d$ up to $k d=20$ and reaches a minimum normalized value of 0.5 when the contact radius becomes half of the radius of the CMUT cell. Similar to the radiation resistance, the radiation reactance peak also shifts to

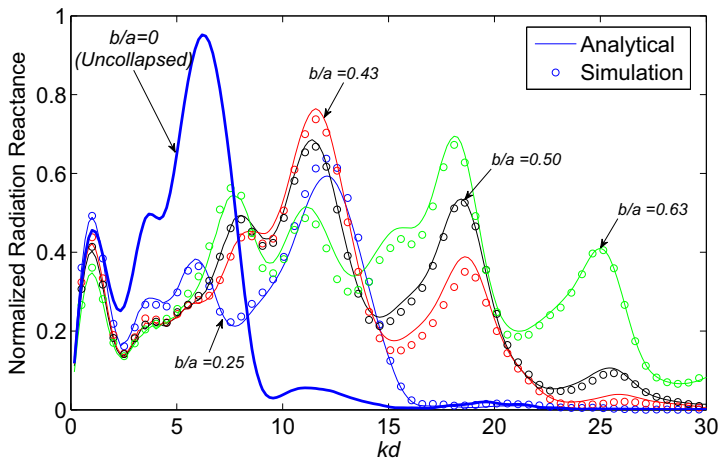

Fig. 6. Normalized radiation reactance for a 7-cell closely packed CMUT array in collapsed state for different $b / a$. For comparison, radiation impedance in uncollapsed regime is also included.

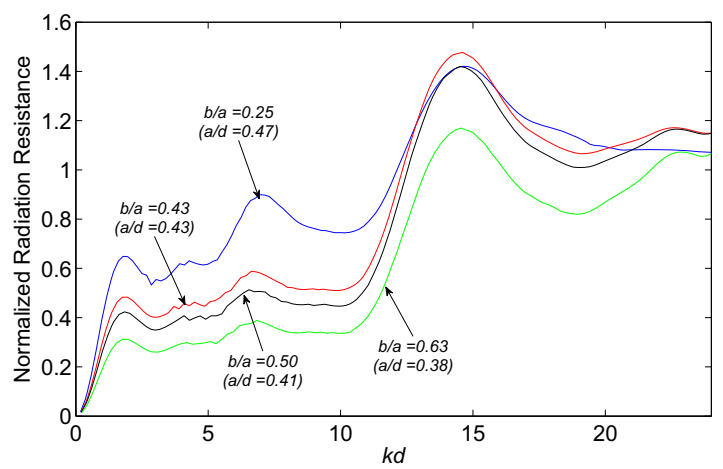

Fig. 7. Normalized maximum radiation resistance results for a 7-cell CMUT array in collapsed state for different $(b / a)$ ratios in optimum $(a / d)$.

higher $k d$ values with a lower peak value in collapsed state in comparison to uncollapsed mode of operation.

As mentioned previously, Figs. 5 and 6 are for closely packed placement. Radiation resistance peak can be further maximized by optimizing the gap between the neighboring cells. Maximized peak radiation resistance results obtained following this approach and optimum $a / d$ values for different $b / a$ ratios can be seen in Fig. 7 and Table I, respectively. At this point, it is worth mentioning why the achievement of a high peak radiation resistance in collapsed state has such an importance. In a recent paper [6], it is shown that a small gap, $t_{g}$ is needed to generate more electrostatic power for transmitting applications. However, a small gap means a limit on the particle velocity. Since the power delivered to the medium is the product of the radiation resistance and the square of the particle velocity, to maximize the delivered power radiation resistance must be maximized.

In the second set of results plotted in Figs. 8 and 9, the contact radius is kept constant at an average value $(b / a=0.37)$ and the radiation impedance in collapsed state is investigated as the number of tiers in the array is changed from two to five, corresponding to 7, 19, 37, and 61 cells. As seen in the figures, the radiation resistance of an array reaches to a plateau of normalized value of 0.5 and maintains this level for a wide $k d$ range from $7.4 d / D$ to 10.6 . Here $D$ represents 


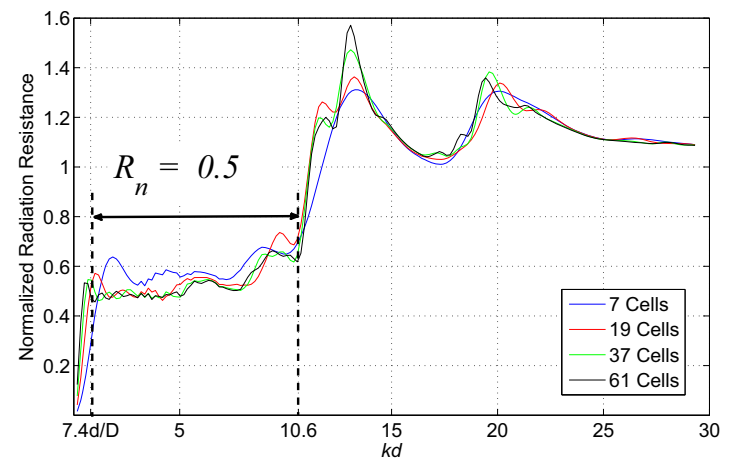

Fig. 8. Analytical normalized radiation resistance for an array of CMUT cells placed in a hexagonal pattern with $7,19,37$, and 61 cells in collapsed state for a fixed $b / a=0.37$ and $a / d=0.50$.

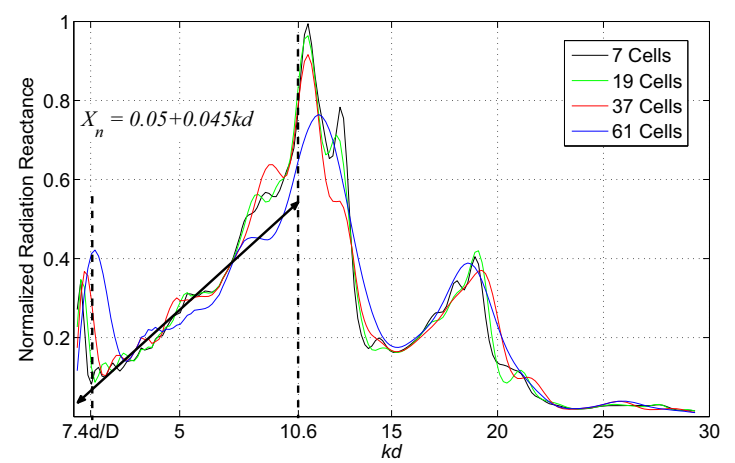

Fig. 9. Analytical normalized radiation reactance for an array of CMUT cells placed in a hexagonal pattern with $7,19,37$, and 61 cells in collapsed state for a fixed $b / a=0.37$ and $a / d=0.50$.

the total diameter of the array as shown in Fig. 4. Hence the normalized radiation resistance in collapsed state for an array can be modelled with a normalized resistance $R_{n}=0.5$ if the $k d$ value is in this range. Notice that for arrays with more cells, the radiation resistance maintains its value for lower $k d$ values. The peak value of radiation resistance is reached about $k d=14.5$ and this peak value increases as the number of cells in the array increases. The variation of the normalized reactance with respect to frequency indicates a constant slope in the aforementioned $k d$ range and can be modelled with a normalized reactance $X_{n}=0.05+0.045 \mathrm{kd}$ for $7.4 d / D<k d<10.6$ where the normalization constant is $\rho S c$.

\section{CONCLUSIONS}

In this work, we first calculate the radiation impedance of a single collapsed CMUT cell. It is found and verified by FEM simulations that as the contact radius increases in collapsed state, the radiation impedance decreases for lower $k a$ values and becomes more oscillatory for the whole $k a$ range. Then, we move on to the radiation impedance of an array of circular CMUT cells in collapsed state. It is understood that for an array of fixed number of cells, the radiation resistance and reactance peaks shift to higher $k d$ values with lower peak values in collapsed state as compared to the conventional mode. In addition, as the contact radius is increased further the radiation resistance becomes smaller for almost all $k d$ values up to $k d=20$. Instead of keeping the number of cells in the array constant, if the contact radius is kept constant and the cell count in the array is changed, it is seen that the radiation resistance and reactance peak values increase with the increasing number of cells, although the location of the peaks is not affected significantly as in the uncollapsed case. For $7.4 d / D<k d<10.6$, it is safe to take the normalized radiation resistance as 0.5 and the normalized radiation reactance as $0.05+0.045 k d$.

\section{REFERENCES}

[1] R. O. Guldiken, J. Zahorian, F. Y. Yamaner, and F. L. Degertekin, "Dual-electrode CMUT with non-uniform membranes for high electromechanical coupling coefficient and high bandwidth operation," IEEE Trans. Ultrason., Ferroelect., Freq. Contr., vol. 56, pp. 1270-1276, 2009.

[2] S. Olcum, F. Y. Yamaner, A. Bozkurt, H. Köymen, and A. Atalar, "An equivalent circuit model for transmitting capacitive micromachined ultrasonic transducers in collapse mode," IEEE Trans. Ultrason., Ferroelect., Freq. Contr., vol. 58, pp. 1468-1477, 2011.

[3] M. N. Senlik, S. Olcum, H. Köymen, and A. Atalar, "Radiation impedance of an array of circular capacitive micromachined ultrasonic transducers," IEEE Trans. Ultrason., Ferroelect., Freq. Contr., vol. 57, pp. 969-976, 2010.

[4] L. Sherman, "Analysis of acoustic interactions in transducer arrays," IEEE Trans. Sonics Ultrason., vol. 13, pp. 9-15, 1966.

[5] M. Greenspan, "Piston radiator: Some extensions of the theory," J. Acoust. Soc. Am., vol. 65, pp. 608-621, 1979.

[6] S. Olcum, F. Y. Yamaner, A. Bozkurt, H. Köymen, and A. Atalar, "Deep collapse operation of capacitive micromachined ultrasonic transducers," IEEE Trans. Ultrason., Ferroelect., Freq. Contr., vol. 58, pp. 2475-2483, 2011. 